\title{
The rs237887 single nucleotide polymorphism in oxytocin receptor gene and the risk for mood disorders in Italian population: A case-control study
}

\author{
Antonio Bruno", Chiara Gangemi\#, Agnese Gugliandolo, Manuela Crucitti, Monica Currò, Clemente Cedro, Gianluca Pandolfo, Daniela \\ Caccamo*, Rocco A. Zoccali and Maria R.A. Muscatello \\ Department of Biomedical Sciences, Dental Sciences and Morpho-functional Imaging, Polyclinic Hospital University of Messina, Messina, Italy \\ \#These authors equally contributed to this work.
}

\begin{abstract}
Primary objective and hypothesis: Oxytocin plays a key role as regulator of several prosocial behaviors, and its plasma and cerebrospinal fluid concentrations have been found to be reduced in psychiatric conditions involving alterations in social phenotypes. Interestingly, single nucleotide polymorphisms (SNPs) in oxytocin receptor (OXTR) gene sequence have been reported to be associated with autism, personality disorder, and depression. Recently, the SNP rs237887 has been reported to lie in a functional region of OXTR gene, required for transcriptional regulation of OXTR, and, as consequence, likely playing a role in oxytocin pathway dysregulation. We aimed to first investigate the potential role of OXTR rs237887 as genetic risk factor for mood disorders.

Research design: We carried out a case-control study by evaluating the distribution of OXTR rs237887 in 87 patients with major depressive disorder (MDD), 128 with bipolar disorder (BD), and 140 healthy subjects, matched for age and gender. Genotyping was performed by Real-time PCR allelic discrimination.

Outcomes and results: A higher frequency for OXTR rs $237887 \mathrm{G}$ mutated allele was found in MDD patients compared with BD patients and controls $(0.48$ vs 0.42 vs 0.42). Moreover, the frequency of GG mutated genotype was higher in MDD than in BD patients and controls ( $24 \%$ vs $17 \%$ vs $16 \%$ ). Instead, similar genotype and allele frequencies were observed between BD cases and controls. However, the differences found were not statistically significant, likely due to the small sample size.

Conclusions: These preliminary observations suggest to move the interest on OXTR rs237887 polymorphism towards MDD and, at the same time, replicate the present results in a larger sample size.
\end{abstract}

\section{Introduction}

Oxytocin (OT) has long been known as the hormone responsible for parturition and lactation. However, recent evidences have shown that OT is a key regulator of various pro-social behaviors, such as social bonding, attachment, love, trust, positive communication, empathy and altruism, and likely plays a role in social reward systems [1,2].

OT is a nonapeptide mainly synthesized in the magnocellular neurons of the paraventricular and supraoptic nuclei of the hypothalamus, and released by the pituitary gland. The oxytocinergic system is involved in the neuromodulation of amygdala, hypothalamus and nucleus accumbens activities, and also crosstalks with monoamine (serotonin, norepinephrine, and dopamine) pathways. These observations suggest a role for OT in the regulation of 'prosocial' behaviors, especially those involving affective components $[3,4]$. Indeed, the OT system dysregulation has been suggested as a putative mechanism contributing to disturbed social interactions and altered social recognition skills [2,5]. Several evidences demonstrate that abnormalities in social cognition in schizophrenia, major depressive disorder (MDD), and bipolar disorder (BD), as well as the neurobiology of social cognition involve a role for OT [6]. Notably, human biomarker studies show that OT concentrations in plasma and cerebrospinal fluid are negatively correlated with measures of aggression, suicidality, and negative emotions after social exclusion [7-9].
Interestingly, social cognitive performance may be strongly influenced by the context, i.e. the quality of early environmental experiences and inter-individual differences [10]. In this regard, previous findings from behavioural studies also suggest that differences in socio-emotional processing are associated with genetic variation in the OXTR (chr. 3p25.3), the gene encoding for OT receptor, in samples of young and older adults [11]. Indeed, polymorphic variants of OXTR not only are associated with impaired social skills [12-15], but also significantly affect the differential susceptibility to psychiatric conditions, including anxiety disorder, autism, personality disorders, and depression [16-19].

Recent evidences suggest a role for OT pathway dysregulation in MDD [20-21]. An altered pattern of peripheral OT release was

Correspondence to: Daniela Caccamo, Department of Biomedical Sciences, Dental Sciences and Morpho-functional Imaging, Polyclinic Hospital University, Via C. Valeria 1, -98125 Messina, Italy, Tel: +39 0902213389, Fax: +390902213382, E-mail: dcaccamo@unime.it

Key words: oxytocin receptor, major depressive disorder, bipolar disorder, gene polymorphisms, OXTR rs 237887

Received: October 13, 2016; Accepted: October 27, 2016; Published: October 30,2016 
observed in depressed women, that showed lower OT plasma levels in comparison with healthy ones [22-23]; moreover, salivary OT levels were lower in mothers with chronic depression and their children compared to non-depressed mothers [24].

Consistently with OT involvement in mood related disorders, previous works have investigated the association between OXTR single nucleotide polymorphisms (SNP) rs53576, rs2254298, rs237885 and depression [18,24-26]. To date, only the rs2254298 resulted to be associated with MDD, since the rs2254298 GG homozygous mutated genotype was over-represented in depressed mothers and their families, and it correlated with lower salivary OT levels [24].

Recent reports described the association of a different OXTR SNP, rs237887, with prosociality and social skills $[14,27]$. This polymorphism lies in intron 3 of OXTR gene, a functional region containing an ETS family target sequence $\left(5^{\prime}\right.$-GGA-3') required for the transcriptional regulation of OXTR gene [14]. These features, suggesting that OXTR rs237887 variant might play a role in OT pathway dysregulation, prompted us to carry out a case-control study investigating, for the first time, the association of OXTR SNP rs237887 with MDD and BD.

\section{Materials and methods}

\section{Study cohort}

This study was carried out on 215 patients with affective disorders that were referring for evaluation to the Psychiatry Unit of the University Hospital of Messina (Italy). Eighty-seven patients were diagnosed with MDD and 128 patients with $\mathrm{BD}$. Consensus life time diagnosis was made for each patient by two experienced psychiatrists. The phenotyping was based on clinical interviews, medical records and family history method according to DSM-IV criteria using SCID (Structured Clinical Interview for DSM Disorders) [28]. All relevant clinical informations regarding the course of both disorders were provided by SCID.

The control group consisted of 140 healthy subjects, matched with patients for age, gender and ethnicity, that were recruited, on a voluntary basis, among the general population as well as staff and students of the University Hospital of Messina. All subjects in control group were psychiatrically screened using the Italian version of MiniInternational Neuropsychiatric Interview - M.I.N.I [29], for excluding the presence of any serious mental health problems.

Written informed consent was individually obtained by each recruited subject, and the study was performed according to the Code of Ethics of the World Medical Association (Declaration of Helsinki).

\section{Genotyping for OXTR rs237887 single nucleotide polymorphism}

Genomic DNA was isolated from peripheral blood lymphocytes by QIAamp DNA Blood Mini Kit (Qiagen, Hilden, Germany) according to the manufacturer's instructions. Then, genotyping for OXTR rs237887 polymorphism (Allele $1=\mathrm{G}$; allele 2=A; genomic coordinates 8772042 , available from National Center for Biotechnology Information dbSNP Build 123) was carried out by Real-time PCR allelic discrimination using a commercially available TaqMan Genotyping Assay (C_3290323_10) (Applied Biosystems, Monza, Italy), and Genotyping Master Mix (Applied Biosystems, Monza, Italy), according to manufacturer's instructions. Samples with OXTR rs237887 known genotype, previously assessed by DNA direct sequencing, were also run as controls for genotyping.

Thermal cycling conditions were set according to manufacturer instructions. Real-time PCR instrument was a Fast 7900 HT Real-time PCR (Applied Biosystems, Monza, Italy).

\section{Statistical analysis}

Data obtained from the study underwent descriptive and inferential statistical analysis. Continuous data were expressed as mean $\pm \mathrm{SD}$, and comparison among the groups was performed using a one-way analysis of variance (one-way ANOVA), followed by the Bonferroni's post hoc test, or Student's $t$ - test, when appropriated. Non-continuous data were expressed as percentages, and the comparison among the groups was performed by using the Chi-Square test. Compliance of genotype distribution to the Hardy-Weinberg equilibrium was estimated by Fisher's exact test, based on a Web program (http://ihg.gsf.de/cgi-bin/ hw/hwal.pl). Statistical analysis wasperformed with GraphPad Prism 5 software (San Diego, CA, USA).

\section{Results}

Clinical and demographic features of study participants are shown in table 1 . At the time of recruitment, MDD and $\mathrm{BD}$ patients and healthy subjects were well matched with regard to and educational level.

Statistical analysis showed that MDD and BD patients were well matched for age and gender, while significant differences were found for educational level and duration of illness, that were higher in the $\mathrm{BD}$ group, and also for age of onset, that was higher in MDD group (Table 1).

The results from genotyping of cases and controls at OXTR locus rs237887 are shown in table 2. Genotype distributions were in HardyWeinberg equilibrium both in cases and controls ( $p=0.8$ for MDD, $\mathrm{p}=1$ for $\mathrm{BD}, \mathrm{p}=0.6$ for controls).

Our data show that the wild-type A allele was more represented than the $G$ mutated allele both in recruited patients and healthy subjects. Interestingly, a lower frequency for A allele, and conversely a higher frequency for $\mathrm{G}$ mutated allele, was found in MDD patients

Table 1. Socio-demographic and clinical features of study subjects.

\begin{tabular}{|l|c|c|c|}
\hline & $\begin{array}{c}\text { MDD } \\
\text { Patients } \\
(\mathbf{n = 8 7 )}\end{array}$ & $\begin{array}{c}\text { BD } \\
\text { Patients } \\
(\mathbf{n = 1 2 8})\end{array}$ & $\begin{array}{c}\text { Controls } \\
(\mathbf{n}=\mathbf{1 4 0})\end{array}$ \\
\hline Age (years) ${ }^{\mathrm{a}}$ & $49.8 \pm 10.7$ & $46.9 \pm 10$ & $48.2 \pm 9.9$ \\
\hline Gender (\% female) $^{b}$ & 47.1 & 39.1 & 38.6 \\
\hline Educational level (years) $^{\mathrm{a}}$ & $10.6 \pm 4.1$ & $11.9 \pm 3.5$ & $11.6 \pm 3.9$ \\
\hline Age of onset (years) $^{c}$ & $43 \pm 15.5$ & $30.5 \pm 10.3$ & - \\
\hline Duration of illness (years) $^{c}$ & $8.1 \pm 8$ & $16 \pm 9.6$ & - \\
\hline
\end{tabular}

Data are provided as means $\pm \mathrm{SD}$, and were analysed by ${ }^{\mathrm{a}} \mathrm{ANOVA},{ }^{\mathrm{b}} \mathrm{Chi}$-Square test; ${ }^{\mathrm{c}}$ Student's $t$-test.

Values were considered statistically significant when $\mathrm{p}<0.05$. 
Table 2. Genotype and allele distributions of OXTR rs237887 polymorphism in patients affected by mood disorders and healthy subjects.

\begin{tabular}{|c|c|c|c|c|c|c|}
\hline $\begin{array}{c}\text { OXTR rs237887 SNP } \\
(\mathrm{A}>\mathrm{G})\end{array}$ & $\begin{array}{l}\text { Controls } \\
(n=140)\end{array}$ & $\begin{array}{l}\text { MDD patients } \\
\quad(\mathbf{n}=\mathbf{8 7})\end{array}$ & $\begin{array}{l}\text { BD patients } \\
(n=128)\end{array}$ & $\mathbf{p}^{\mathrm{a}}$ & $\mathbf{p}^{\mathrm{b}}$ & $\mathbf{p}^{\mathrm{c}}$ \\
\hline $\mathbf{A} / \mathbf{A}$ & $32.1 \%(45)$ & $27.6 \%(24)$ & $32.8 \%(42)$ & 0.47 & 0.91 & 0.41 \\
\hline $\mathbf{A} / \mathbf{G}$ & $51.4 \%(72)$ & $48.3 \%(42)$ & $50 \%(64)$ & 0.64 & 0.88 & 0.80 \\
\hline G/G & $16.4 \%(23)$ & $24.1 \%(21)$ & $17.2 \%(22)$ & 0.153 & 0.86 & 0.21 \\
\hline$A$ allele frequency & 0.58 & 0.52 & 0.58 & 0.20 & 0.99 & 0.21 \\
\hline G allele frequency & 0.42 & 0.48 & 0.42 & 0.20 & 0.99 & 0.21 \\
\hline
\end{tabular}

${ }^{a} \mathrm{p}$ value obtained after comparison of MDD and Ctr; ${ }^{b} \mathrm{p}$ value obtained after comparison of BD and Ctr; ${ }^{\mathrm{c}} \mathrm{p}$ value obtained after comparison of MDD and BD. Values were considered statistically significant when $\mathrm{p}<0.05$.

than in BD and controls (Table 2). However, these differences were not significant.

The A/G heterozygous genotype was the most represented in all groups, with similar frequencies, while the G/G mutated genotype was more frequent in patients than in healthy subjects, with a higher frequency in MDD group than in BD group. However, all these differences did not reach statistically significant values. Conversely, the frequency of A/A wild-type genotype was slightly lower in MDD patients (Table 2).

\section{Discussion}

Interest in the role of OT in mood disorders has increased with the demonstration that this hormone plays an important role in social attraction, affiliative behavior and bonding, which might be important in relation to the development of depression [10,30]. Since OT can decrease anxiety levels and, more in general, the endocrine response to environmental stressors, as well as modulate cognitive functions and promote positive social relationships, some symptoms of depression, including social withdrawal, cognitive impairment, appetite modifications and stress reactivity may be influenced by dysregulation of the OT system [21,31]. In this regard, intranasal OT administration has been associated with reduced social stress reactivity, and attenuated amygdala activity in response to emotional stimuli [32].

Notably, it has been shown that individual differences in OXTR gene sequence may strongly influence individual differences in social cognition [14]. Since these results have translational potential in depression, social stress, and anxiety, they could increase the understanding of the role of OT in aging, in which impairment of socio-emotional function is often observed, and will have the potential for generating new interventions to improve health and well-being [11].

To date, most studies investigating the relationship between OXTR and social phenotypes have focused primarily on SNPs rs1042778 [33], rs53576 [34], and rs2254298 [35]. Israel and co-workers [33] testing OXTR genetic polymorphisms for association with the Dictator Game and a related paradigm, the Social Values Orientation task, confirmed the relation between oxytocin system and human prosocial decision. Moreover, Chen and co-workers [34] investigated the possible interaction between the SNP rs53576 in OXTR gene with stressprotective effects of social support. Given that individuals with one or two copies of the $G$ allele of rs53576 showed lower cortisol responses to stress after social support in comparison with subjects with the same genotype receiving no social support, their findings indicate that genetic variation of the oxytocin system could modulate the effectiveness of positive social interaction. Interestingly, Tost and co-workers [35] demonstrated that the OXTR rs2254298 SNP has neurogenetic effects, as it affects amygdala volume in healthy Caucasian subjects.
Previous works have investigated the association between depression and OXTR rs53576, rs2254298, rs237885 [18,24,26,36], showing that only the rs 2254298 is associated with MDD, and correlates with lower salivary OT levels in depressed mothers and their families [24].

Another polymorphism, rs237887, has been reported to lie in a block located in intron 3 of the OXTR gene, that contains three other SNPs (rs237885, rs11706648, rs2268490) in moderate linkage disequilibrium (highest $\mathrm{r}^{2}=0.74$, between $\mathrm{rs} 237887$ and $\mathrm{rs} 237885$ ). This is a functionally relevant region of the OXTR gene, since it has been shown that a specific motif of 10-15 nucleotides close to the middle of intron 3 specifically binds nuclear proteins correlating with the down-regulated state of the gene. The accumulated data suggest that this intronic element may be involved in transcriptional regulation of OXTR gene expression [4,37].

Interestingly, the rs237887 SNP has been associated with different neurological conditions, such as empathy, schizophrenia, and impairment of human social recognition skills $[14,38,39]$. Wu and coworkers [39], in a Chinese population, correlated rs237887 genotypes with four subscales of the Interpersonal Reactivity Index, in particular with personal distress, empathy, perspective taking and fantasy. They observed that in personal distress, individuals with GG genotype scored higher than heterozygous AG subjects, while in fantasy, those with GG genotype showed a higher score than people with AA and AG genotype. Moreover, individuals with A allele had a higher level of perspective taking than those with $\mathrm{G}$ allele. Concerning emotional empathy, they observed that the $G$ allele carriers displayed more emotional empathy than Aallele ones.

Skuse and co-workers [14] found a strong association between rs237887 SNP and recognition memory in combined probands, parents, and siblings, suggesting a critical role for the oxytocin system in social recognition.

OXTR rs237887 SNP has also been associated with emotional and behavioral reactions to betrayal. The wild-type allele A-rs237887 was associated with higher levels of post-betrayal satisfaction, while the mutated allele G-rs237887 resulted associated with lower levels of post-betrayal satisfaction [40]. Recent investigations suggest the involvement of several OXTR SNP, including rs237887, in autism spectrum disorder [41], and in individual phenotypic differences in pro-sociality, in association with the clock genes [27].

OXTR rs237887 has been reported to be associated with several cognitive processes and prosocial behavior; however, to date, no data are available concerning its potential link with mood related disorders. Thus, we aimed to investigate the relationship between mood disorders (MDD, BD) and this OXTR variant, by analyzing the differences in allele and genotype frequencies between patients and healthy subjects. Although we did not find any significant association of OXTR rs237887 
with mood disorders, interestingly we observed that the $\mathrm{G}$ minor allele, as well as the GG mutated genotype, showed a higher frequency in MDD patients compared with BD patients and controls. Even if these differences did not reach the statistical significance, likely due to the small size of affected populations recruited for this study, they suggest a potential involvement of OXTR rs237887 SNP in the alteration of OT system in MDD patients. Moreover, the similar allele and genotype frequencies found between $\mathrm{BD}$ patients and controls, suggest that $\mathrm{BD}$ patients, in this regard, are genetically more similar to healthy subjects than MDD ones. Unfortunately, there are not available literature data about the distribution of OXTR rs237887 SNP in either the Caucasian general population or MDD and BD patients. Thus, we were not able to compare our results with previously reported observations in people of the same ethno-geographic area. However, genotype frequencies observed in our control group were similar to those found by Tabak and co-workers [40] in an American population composed by nonHispanic White individual. Interestingly, rs237887 minor allele and genotype frequencies found in our BD patients and healthy subjects were similar to those previously reported in an investigation carried out in a Chinese population of 498 individuals by Kawamura and coworkers [16]. These authors reported the lack of association between rs237887 and affective temperaments (depressive, cyclothymic, hyperthymic, irritable, anxious), even if they stated that their study had some limitations, given that participants were from a corporation, not from the general community, possibly having a different TEMPS-A distribution.

Given that only MDD patients show different allelic and genotypic differences compared to control subjects, our data highlight the necessity to move the interest on OXTR rs237887 polymorphism towards MDD and, at the same time, replicate the present results in a larger population. In fact, our study was insufficiently powered to detect the true association of this OXTR SNP with MDD due to the sample size, that was small for a case-control study. Moreover, given the observed differences in genotype frequencies between MDD and BD patients, it would be worthy to elucidate whether OXTR rs 237887 polymorphism might represent a diagnostic tool able to discriminate between these two mood disorders.

It has been demonstrated a role for OT to influence neural plasticity in response to environmental circumstances, and 'for better or for worse', and a potential ability to affect later behavioral and psychological outcomes [42]. Notably, even if OXTR rs237887 polymorphism has been associated with many aspects of social cognition as well as several prosocial behaviors, so far it has not been investigated in mood related disorders. Moreover, since this polymorphism could affect OXTR expression, further studies evaluating the relationships between OXTR expression levels and the individual OXTR genetic background will be helpful to elucidate the neuromodulator role of OT in brain regions of MDD patients, in which disturbed social interactions are often apparent.

\section{Author contributions}

$A B, M R A M$, and RAZ established selection criteria of patients; CC, GP, and MC recruited patients for the study; MC recruited control subjects for the study; CG carried out genetic analyses; AG carried out statistical analyses; $C G$ and $A B$ participated in manuscript drafting; MRAM participated in study conception and design, and revised the manuscript critically; DC conceived and designed the work, coordinated experimental work, performed data interpretation, drafted the manuscript, and gave final approval of the version to be published.
All authors read and approved the final manuscript.

\section{References}

1. Macdonald K, Macdonald TM (2010) The peptide that binds: a systematic review of oxytocin and its prosocial effects in humans. Harv Rev Psychiatry 18: 1-21. [Crossref]

2. Shamay-Tsoory SG, Abu-Akel A (2016) The Social Salience Hypothesis of Oxytocin. Biol Psychiatry 79: 194-202. [Crossref]

3. Ross HE, Young LJ (2009) Oxytocin and the neural mechanisms regulating social cognition and affiliative behavior. Front Neuroendocrinol 30: 534-547. [Crossref]

4. Baskerville TA, Douglas AJ (2010) Dopamine and oxytocin interactions underlying behaviors: potential contributions to behavioral disorders. CNS Neurosci Ther 16: e92123. [Crossref]

5. Guastella AJ, Mitchell PB, Dadds MR (2008) Oxytocin increases gaze to the eye region of human faces. Biol Psychiatry 63: 3-5. [Crossref]

6. Mercedes Perez-Rodriguez M, Mahon K, Russo M, Ungar AK, Burdick KE (2015) Oxytocin and social cognition in affective and psychotic disorders. Eur Neuropsychopharmacol 25: 265-282. [Crossref]

7. Lee R, Ferris C, Van de Kar LD, Coccaro EF (2009) Cerebrospinal fluid oxytocin, life history of aggression, and personality disorder. Psychoneuroendocrinology 34: 15671573. [Crossref]

8. Jokinen J, Chatzittofis A, Hellström C, Nordström P, Uvnäs-Moberg K, et al. (2012) Low CSF oxytocin reflects high intent in suicide attempters. Psychoneuroendocrinology 37 : 482-490. [Crossref]

9. Jobst A, Sabass L, Palagyi A, Bauriedl-Schmidt C, Mauer MC, et al. (2015) Effects of social exclusion on emotions and oxytocin and cortisol levels in patients with chronic depression. J Psychiatr Res 60: 170-177. [crossref]

10. Olff M, Frijling JL, Kubzansky LD, Bradley B, Ellenbogen MA, et al. (2013) The role of oxytocin in social bonding, stress regulation and mental health: an update on the moderating effects of context and interindividual differences. Psychoneuroendocrinology 38: 1883-1894. [Crossref]

11. Ebner NC, Maura GM, Macdonald K, Westberg L, Fischer H (2013) Oxytocin and socioemotional aging: Current knowledge and future trends. Front Hum Neurosci 7 : 487. [Crossref]

12. Rodrigues SM, Saslow LR, Garcia N, John OP, Keltner D (2009) Oxytocin receptor genetic variation relates to empathy and stress reactivity in humans. Proc Natl Acad Sci US A 106: 21437-21441. [Crossref]

13. Walum H, Lichtenstein P, Neiderhiser JM, Reiss D, Ganiban JM, et al. (2012) Variation in the oxytocin receptor gene is associated with pair-bonding and social behavior. Biol Psychiatry 71: 419-426. [Crossref]

14. Skuse DH, Lori A, Cubells JF, Lee I, Conneely KN, et al. (2014) Common polymorphism in the oxytocin receptor gene (OXTR) is associated with human social recognition skills. Proc Natl Acad Sci U S A 111: 1987-1992. [Crossref]

15. Slane MM, Lusk LG, Boomer KB, Hare AE, King MK, et al. (2014) Social cognition, face processing, and oxytocin receptor single nucleotide polymorphisms in typically developing children. Dev Cogn Neurosci 9:160-171. [Crossref]

16. Kawamura Y, Liu X, Akiyama T, Shimada T, Otowa T, et al. (2010) The association between oxytocin receptor gene (OXTR) polymorphisms and affective temperaments, as measured by TEMPS-A. J Affect Disord 127: 31-37. [Crossref]

17. Liu X, Kawamura Y, Shimada T, Otowa T, Koishi S, et al. (2010) Association of the oxytocin receptor (OXTR) gene polymorphisms with autism spectrum disorder (ASD) in the Japanese population. J Hum Genet 55: 137-141. [Crossref]

18. Brüne M (2012) Does the oxytocin receptor (OXTR) polymorphism (rs2254298) confer 'vulnerability' for psychopathology or 'differential susceptibility'? Insights from evolution. BMC Med 10: 38. [Crossref]

19. Myers AJ, Williams L, Gatt JM, McAuley-Clark EZ, Dobson-Stone C, et al. (2014) Variation in the oxytocin receptor gene is associated with increased risk for anxiety, stress and depression in individuals with a history of exposure to early life stress. $J$ Psychiatr Res 59: 93-100. [Crossref]

20. ScantamburloG,HansenneM,FuchsS,PitchotW,MaréchalP,etal.(2007)Plasmaoxytocin levels and anxiety in patients with major depression. Psychoneuroendocrinology 32 : 407-410. [Crossref]

21. McQuaid RJ, McInnis OA, Abizaid A, Anisman H (2014) Making room for oxytocin in understanding depression. Neurosci Biobehav Rev 45: 305-322. [Crossref] 
22. Cyranowski JM, Hofkens TL, Frank E, Seltman H, Cai HM, et al. (2008) Evidence of dysregulated peripheral oxytocin release among depressed women. Psychosom Med 70: 967-975. [Crossref]

23. Yuen KW, Garner JP, Carson DS, Keller J, Lembke A, et al. (2014) Plasma oxytocin concentrations are lower in depressed vs. healthy control women and are independent of cortisol. J Psychiatr Res 51: 30-36. [Crossref]

24. Apter-Levy Y, Feldman M, Vakart A, Ebstein RP, Feldman R (2013) Impact of maternal depression across the first 6 years of life on the child's mental health, social engagement, and empathy: the moderating role of oxytocin. Am J Psychiatry 170: 1161-1168. [Crossref]

25. Thompson RJ, Parker KJ, Hallmayer JF, Waugh CE, Gotlib IH (2011) Oxytocin receptor gene polymorphism (rs2254298) interacts with familial risk for psychopathology to predict symptoms of depression and anxiety in adolescent girls. Psychoneuroendocrinology 36: 144-147. [Crossref]

26. Mileva-Seitz V, Steiner M, Atkinson L, Meaney MJ, Levitan R, et al. (2013) Interaction between oxytocin genotypes and early experience predicts quality of mothering and postpartum mood. PloS One 8: e61443. [Crossref]

27. Ci H, Wu N, Su Y (2014) Clock gene modulates roles of OXTR and AVPR1b genes in prosociality. PLoS One 9: e109086. [Crossref]

28. First MB, Donovan S, Frances A (1996) Nosology of chronic mood disorders. Psychiatr Clin North Am 19: 29-39. [Crossref]

29. Rossi A, Alberio R, Porta A, Sandri M, Tansella M, et al. (2004) The reliability of the Mini-International Neuropsychiatric Interview--Italian version. $J$ Clin Psychopharmacol 24: 561-563. [Crossref]

30. Neumann ID (2008) Brain oxytocin: a key regulator of emotional and social behaviours in both females and males. J Neuroendocrinol 20: 858-865. [Crossref]

31. Bell CJ, Nicholson H, Mulder RT, Luty SE, Joyce PR (2006) Plasma oxytocin levels in depression and their correlation with the temperament dimension of reward dependence. J Psychopharmacol 20: 656-660. [Crossref]
32. Wudarczyk OA, Earp BD, Guastella A, Savulescu J (2013) Could intranasal oxytocin be used to enhance relationships? Research imperatives, clinical policy, and ethical considerations. Curr Opin Psychiatry 26: 474-484. [Crossref]

33. Israel S, Lerer E, Shalev I, Uzefovsky F, Riebold M, et al. (2009) The oxytocin receptor (OXTR) contributes to prosocial fund allocations in the dictator game and the social value orientations task. PLoS One 4: e5535. [Crossref]

34. Chen FS, Kumsta R, von Dawans B, Monakhov M, Ebstein RP, et al. (2011) Common oxytocin receptor gene (OXTR) polymorphism and social support interact to reduce stress in humans. Proc Natl Acad Sci U S A 108: 19937-19942. [Crossref]

35. Tost H, Kolachana B, Verchinski BA, Bilek E, Goldman AL, et al. (2011) Neurogenetic effects of OXTR rs2254298 in the extended limbic system of healthy Caucasian adults. Biol Psychiatry 70: e37-39. [Crossref]

36. Costa B, Pini S, Gabelloni P, Abelli M, Lari L, et al. (2009) Oxytocin receptor polymorphisms and adult attachment style in patients with depression. Psychoneuroendocrinology 34: 1506-1514. [Crossref]

37. Mizumoto Y, Kimura T, Ivell R (1997) A genomic element within the third intron of the human oxytocin receptor gene may be involved in transcriptional suppression. Mol Cell Endocrinol 135: 129-138. [Crossref]

38. Wu N, Li Z, Su Y (2012) The association between oxytocin receptor gene polymorphism (OXTR) and trait empathy. J Affect Disord 138: 468-472. [Crossref]

39. Souza RP, de Luca V, Meltzer HY, Lieberman JA, Kennedy JL (2010) Schizophrenia severity and clozapine treatment outcome association with oxytocinergic genes. Int $J$ Neuropsychopharmacol 13: 793-798. [Crossref]

40. Tabak BA, McCullough ME, Carver CS, Pedersen EJ, Cuccaro ML (2014) Variation in oxytocin receptor gene (OXTR) polymorphisms is associated with emotional and behavioral reactions to betrayal. Soc Cogn Affect Neurosci 9: 810-816. [Crossref]

41. LoParo D, Waldman ID (2015) The oxytocin receptor gene (OXTR) is associated with autism spectrum disorder: a meta-analysis. Mol Psychiatry 20: 640-646. [Crossref]

42. Belsky J, Jonassaint C, Pluess M, Stanton M, Brummett B, et al. (2009) Vulnerability genes or plasticity genes? Mol Psychiatry 14: 746-754. [Crossref]

Copyright: (C2016 Bruno A. This is an open-access article distributed under the terms of the Creative Commons Attribution License, which permits unrestricted use, distribution, and reproduction in any medium, provided the original author and source are credited. 